\title{
Shapovalov Wave-Like Spacetimes
}

\author{
Konstantin Osetrin ${ }^{1,2, *(\mathbb{C})}$ and Evgeny Osetrin ${ }^{1}(\mathbb{C}$ \\ 1 Theoretical Physics Department, Tomsk State Pedagogical University, 634061 Tomsk, Russia; zyxel@tspu.edu.ru \\ 2 Theoretical Physics Department, Tomsk State University, 634050 Tomsk, Russia \\ * Correspondence: osetrin@tspu.edu.ru
}

Received: 23 July 2020; Accepted: 15 August 2020; Published: 18 August 2020

check for updates

\begin{abstract}
A complete classification of space-time models is presented, which admit the privileged coordinate systems, where the Hamilton-Jacobi equation for a test particle is integrated by the method of complete separation of variables with separation of the isotropic (wave) variable, on which the metric depends (wave-like Shapovalov spaces). For all types of Shapovalov spaces, exact solutions of the Einstein equations with a cosmological constant in vacuum are found. Complete integrals are presented for the eikonal equation and the Hamilton-Jacobi equation of motion of test particles.
\end{abstract}

Keywords: gravity; Hamilton-Jacobi equation; eikonal equation; Killing fields; separation of variables; Stackel spaces; Shapovalov spaces; gravitational waves

\section{Introduction}

The last 30 years have brought great advances in gravity and cosmology in connection with the discovery of the accelerated expansion of the Universe and the discovery of gravitational waves. The phenomenon of "dark energy" and "dark matter", which arose when trying to theoretically explain the accelerated expansion of the Universe within the framework of the general theory of relativity (GR), the phenomenon of cosmological constant-all these things require theoretical understanding and the construction of theoretical models that correspond to observational data, and also obtaining and the additional observational data themselves for the selection of viable theoretical "constructions". Modified theories of gravity are often used as theoretical models explaining the phenomenon of "dark energy" and "dark matter". Furthermore, new observational data have now begun to arrive in the framework of a new direction of research—gravitational-wave astronomy, which arose after the experimental discovery of gravitational waves.

This work is in line with attempts to obtain additional mathematical tools both in the field of methods of gravitational wave astronomy and in the field of studying and comparing modified theories of gravity and general relativity. One of the constructive mathematical methods for constructing exactly integrable models in the theory of gravity and cosmology is the theory of Stäckel spaces, which admit integration of the equations of motion of test particles and radiation by the method of complete separation of variables in the Hamilton-Jacobi equation. Note that the classes of Stäckel spaces include most of the known exact solutions in general relativity-the Schwarzschild metric, Kerr metric, Friedman-Robertson-Walker metric, Kasner metric, NUT metric, etc.

As it became clear recently (in the 1970s-1980s), the possibility of separating variables in the equation of motion of test particles in a gravitational field is rigidly connected with the presence of spacetime symmetries through the so-called "complete sets" of Killing vector and tensor fields, which correspond to additional algebraic conditions. Among such spaces, models that allow the separation of isotropic (null) 
variables, on which the spacetime metric depends, are currently of great interest. We call such models wave-like Shapovalov spaces. In this paper, we will list all types of such spaces. Examples of particular exact gravitational-wave solutions related to these spaces are the solutions obtained earlier in the works of Robertson, Bondi, Trautman, Pirani (see [1]).

Thus, the special classes of spaces proposed for consideration in this work are interpreted as gravitational waves and can serve as "frame" exactly integrable models for gravitational wave astronomy, as well as serve as a theoretical tool for the comparative study of gravitational wave models in modified theories of gravity and in General relativity based on a unified approach, including the formation of a spectrum of observational problems for gravitational wave astronomy.

In this paper, we consider spacetime models that allow integration by the method of complete separation of variables of the eikonal equation and the Hamilton-Jacobi equation for the motion of test particles in a gravitational field, which allow the "privileged" coordinate systems (where separation of variables is possible), in which one of the variables is isotropic (wave).

Spaces that allow complete separation of variables in the Hamilton-Jacobi equation for test particles are called Stäckel spaces after Paul Stäckel, who first posed this problem (Paul Stäckel, see [2]). In a series of papers, Stäckel solved this problem for the case when the metric of space in a privileged coordinate system has a diagonal form. The theory of Stäckel spaces was developed by many authors and was finally completed in the period 1973-1980 in the works of V.N. Shapovalov, who was the first to build a complete classification of these spaces and obtain a general form of their metrics in privileged coordinate systems, where complete separation of variables is allowed (see [3-9]). We recall some details from the theory of Stäckel spaces (for a more complete presentation, see [9-11]).

Let us consider the Hamilton-Jacobi equation for the motion of a test particle of mass $m$ in a gravitational field defined by the metric tensor $g_{i j}$ in the coordinate system $\left\{x^{i}\right\}$ :

$$
g^{i j} \frac{\partial S}{\partial x^{i}} \frac{\partial S}{\partial x^{j}}=m^{2}, \quad i, j, k=1 \ldots n,
$$

where the capital letter $S$ denotes the action function of a test particle, $n$ is the dimension of space. To avoid confusion, note that in what follows we will also use the lowercase $s$ to denote a spacetime interval.

Definition 1. If the space admits the existence of a privileged coordinate system $\left\{x^{i}\right\}$, where the Hamilton-Jacobi Equation (1) admits complete separation of variables, when the complete integral for the action function of the test particle $S$ can be written as:

$$
\begin{aligned}
S=\phi_{1}\left(x^{1}, \lambda_{1}, \ldots, \lambda_{n}\right)+ & \phi_{2}\left(x^{2}, \lambda_{1}, \ldots, \lambda_{n}\right)+\ldots+\phi_{n}\left(x^{n}, \lambda_{1}, \ldots, \lambda_{n}\right), \\
& \lambda_{1}, \ldots, \lambda_{n}-\text { const }, \\
& \operatorname{det}\left|\frac{\partial^{2} S}{\partial x^{i} \partial \lambda_{j}}\right| \neq 0,
\end{aligned}
$$

then such a space is called Stäckel space, and the parameters $\lambda_{j}$ are the constants of separation.

Definition 2. Space with metric $\tilde{g}^{i j}$, allowing complete separation of variables in the eikonal equation for radiation propagation (where $\Psi$ is the eikonal function):

$$
\tilde{g}^{i j} \Psi_{, i} \Psi_{, j}=0,
$$

is called a conformal Stäckel space. 
Conformal Stäckel spaces admit separation of variables in the same privileged coordinate system as a Stäckel spaces, and the metrics of such spaces, as can be seen, differ from the metrics of Stäckel spaces by an arbitrary conformal factor.

The following theorem was proved by V.N. Shapovalov (see [6,9]):

Theorem 1. Let $V_{n}$ be a Stäckel space. Then the components of the metric tensor $g^{i j}$ in a privileged coordinate system can be written as

$$
\begin{gathered}
g^{i j}=\left(\Phi^{-1}\right)_{n}^{v} G_{v}^{i j}, \quad G_{v}^{i j}=G_{v}^{i j}\left(u^{v}\right), \quad \Phi_{\mu}^{v}=\Phi_{\mu}^{v}\left(u^{\mu}\right), \\
G_{v}^{i j}\left(u^{v}\right)=\delta_{v}^{i} \delta_{v}^{j} \varepsilon_{v}\left(u^{v}\right)+\left(\delta_{v}^{i} \delta_{p}^{j}+\delta_{v}^{j} \delta_{p}^{i}\right) G_{v}^{v p}\left(u^{v}\right)+\delta_{p}^{i} \delta_{q}^{j} G_{v}^{p q}\left(u^{v}\right),
\end{gathered}
$$

(there is no summation over index $v$ in Equation (5),

$$
p, q=1, \ldots N, \quad v, \mu=N+1, \ldots n,
$$

where $\Phi_{\mu}^{v}\left(u^{\mu}\right)$ is the so-called Stäckel matrix.

Summation over repeated superscripts and subscripts is accepted, except for the Equation (5). The $N$ is the number of "ignored" variables that the metric in the privileged coordinate system does not depend on. The subscripts $p, q$ number "ignored" variables, and the indices $v, \mu$ number "nonignored" variables.

It is shown that the equation of geodesics in Stäckel spaces admits first integrals that commute pairwise with respect to Poisson brackets

$$
\begin{aligned}
\underset{\mu}{X}=\left(\Phi^{-1}\right)_{\mu}^{v} H_{v}, \quad H_{v} & =\varepsilon_{v} p_{v}^{2}+2 G_{v}^{v p} p_{p} p_{v}+h_{v}^{p q} p_{p} p_{q}, \\
\underset{p}{Y} & =\underset{p}{Y^{i}} p_{i} .
\end{aligned}
$$

Thus, for the covariant characteristic of the Stäckel space, it suffices to find the corresponding properties of the integrals Equations (6) and (7) in an arbitrary coordinate system $\left\{x^{i}\right\}_{n}$.

Let us write the functions $X_{v}, Y_{p}$ in the form

$$
\underset{v}{X}=\underset{v}{X}{ }^{i j} p_{i} p_{j}, \quad \underset{p}{Y}=\underset{p}{Y^{i}} p_{i} .
$$

Then for $X_{v}{ }^{i j}$ and $Y_{p}{ }^{i}$ we get

$$
\underset{v}{X}(i ; k)=\underset{p}{Y}(i ; j)=0,
$$

where semicolon means covariant derivative and parentheses mean symmetrization.

Therefore, $Y_{p}{ }^{i}, X_{v}{ }^{i j}$ are the components of the Killing vector and Killing tensor fields, respectively.

Definition 3. Pairwise commuting Killing vector fields $Y_{p}{ }^{i}$, where $p=1, \ldots, N$ and the Killing tensor fields of the second rank $X_{v}{ }^{i j}$, where $v=N+1, \ldots, n$ form a "complete set" of type $\left(N . N_{0}\right)$ if the following conditions are met:

$$
\begin{gathered}
B^{p q} \underset{p}{\Upsilon_{q}^{i}} \underset{q}{Y^{j}}+B^{v} \underset{v}{X^{i j}}=0 \Longrightarrow B^{p q}=B^{v}=0, \\
\operatorname{rank}\left\|\underset{p}{Y^{i}} \underset{q}{Y_{i}}\right\|=N-N_{0},
\end{gathered}
$$




$$
\begin{aligned}
& \underset{v}{X}{ }^{i k} \underset{\mu}{X}{ }_{k}^{j}=C_{v \mu}^{p q} \underset{p}{\Upsilon_{p}^{i}} \underset{p}{Y^{j}}+C_{\nu \mu}^{\tau} \underset{\tau}{X}{ }^{i j}, \\
& \underset{v}{X_{p}^{i j}} \underset{p}{Y_{j}}=C_{v p}^{q} \underset{q}{Y^{i}} \text {. }
\end{aligned}
$$

Theorem 2. A necessary and sufficient geometric criterion for the Stäckel space is the existence of a complete set of type (N.N).

This theorem was proved by V.N. Shapovalov in the work [9]. Thus, the Hamilton-Jacobi equation can be integrated by the method of complete separation of variables if and only if there is a complete set of first integrals of motion.

You can define the type of Stäckel space as follows:

Definition 4. Spacetime is called a Stäckel space of the type (N.N) if there is a complete set of type (N.N $)$.

All these theorems and definitions are valid if the free Hamilton-Jacobi equation for the motion of test particles in a gravitational field is considered.

Stäckel spaces are determined by the presence of the so-called "complete set" of commuting Killing fields of the first and second rank, corresponding to an additional set of algebraic requirements. Moreover, the $N$ commuting Killing vectors (where $0 \leq N \leq n$ ) included in the "complete set" determine the choice of a "privileged" coordinate system where separation of variables is allowed and where the metric does not depend on the corresponding $N$ variables.

Definition 5. Coordinate variables of the privileged coordinate system, on which the spacetime metric does not depend, are called ignored variable.

Stäckel and conformal Stäckel spaces are of great interest for metric theories of gravity, since they allow explicitly, in quadratures, to integrate the equations for the motion of test particles and for radiation propagation and, thereby, determine the form of geodesic lines of spacetime along which test particles move in gravitational field. The application of these mathematical tools is possible in various metric theories of gravity, including modified theories of gravity with various types of matter (see [12-25]).

\section{Shapovalov Spaces}

The space of the orbits of the $N$-parametric Abelian group of motions of the Stäckel space defined by the Killing vectors from the "complete set" can be "isotropic" in the sense that the restriction of the metric to these orbits can have a determinant equal to zero. Such spaces were first found and classified by V.N. Shapovalov, and he called them "isotropic Stäckel spaces".

If the Killing vectors are from the "complete set" $Y_{(p)}^{i}$, where $p$ numbers the Killing vectors in the set $(p, q=1, \ldots, N)$, then for isotropic Stäckel spaces in the case of four-dimensional spacetime, we obtain $\operatorname{rank}\left|Y_{(p)}^{i} g_{i j} Y_{(p)}^{j}\right|=(N-1)$, that is, the space of the orbits of this group forms an isotropic surface.

The results of Vladimir Shapovalov made it possible for the first time to carry out a complete classification of all spaces admitting the integration of the Hamilton-Jacobi equation of test particles by the method of complete separation of variables, for which in the "privileged" coordinate systems one of the "separated" nonignored variables is the "wave" (otherwise null or isotropic) and find an explicit form of their metrics. We will call such spaces wave-like "Shapovalov spaces" (more precise definitions are given below). 
Definition 6. We will call the coordinate variable isotropic (null), if along the coordinate line of this variable the spacetime interval is equal to zero.

Definition 7. A spacetime that admits complete separation of variables in the Hamilton-Jacobi Equation (1) for a test particle in a gravitational field will be called Shapovalov space if in a privileged coordinate system where separation of variables is allowed, there is a nonignored isotropic variable.

Definition 8. A spacetime admitting complete separation of variables in the eikonal Equation (3) will be called conformal Shapovalov space if there is a nonignored isotropic variable in a privileged coordinate system where separation of variables is allowed.

In total, for the four-dimensional spacetime, there are three main classes of Shapovalov spaces in accordance with the number of commuting Killing vectors in the complete set (from one to three vectors). The form of the metrics in the privileged coordinate system is shown below (further, coordinates are numbered starting from 0 ).

As an example of the application of Shapovalov's wave-like spaces in metric theories of gravity, solutions of the Einstein vacuum equations with the cosmological constant $\Lambda$ are obtained:

$$
R_{i j}-\frac{1}{2} R g_{i j}=\Lambda g_{i j}, \quad \Lambda=\text { const. }
$$

The obtained solutions of the field equations for Shapovalov's wave-like spaces, when the eikonal equation and the Hamilton-Jacobi equation admit the separation of isotropic variables on which the spacetime metric depends, can be interpreted as gravitational waves. Comparative analysis of exact gravitational-wave models for Shapovalov spaces in modified theories of gravity [26-31]) provides an additional tool for comparing and selecting viable theories of modified gravity.

Note that some of the results below could have previously been presented in some form in our other works and in the works of other researchers. The purpose of this work is to give a systematic presentation of the topic under consideration, therefore, all the necessary results that we obtained ourselves are included here.

\section{Type I Shapovalov Spaces}

Shapovalov spaces of type I admit one Killing vector and three Killing tensors of the second rank in the complete set. The metric of the Shapovalov space of type I in the privileged coordinate system $\left\{x^{i}\right\}$ can be written in the following form:

$$
\begin{gathered}
g^{i j}=\frac{1}{\Delta}\left(\begin{array}{cccc}
0 & V^{(1)} & 0 & 0 \\
V^{(1)} & 0 & 0 & 0 \\
0 & 0 & V^{(2)} & 0 \\
0 & 0 & 0 & V^{(3)}
\end{array}\right), \\
V^{(1)}=t_{2}\left(x^{2}\right)-t_{3}\left(x^{3}\right), \quad V^{(2)}=t_{3}\left(x^{3}\right)-t_{1}\left(x^{1}\right), \quad V^{(3)}=t_{1}\left(x^{1}\right)-t_{2}\left(x^{2}\right) .
\end{gathered}
$$

Here, in the case of conformal Shapovalov spaces, $\Delta$ is an arbitrary function of all variables, and for Shapovalov spaces, the conformal factor is $\Delta=\sigma_{1}\left(x^{1}\right) V^{(1)}+\sigma_{2}\left(x^{2}\right) V^{(2)}+\sigma_{3}\left(x^{3}\right) V^{(3)}$. The variable $x^{0}$ is an ignored variable and $x^{1}$ is nonignored isotropic wave variable. 
The spacetime interval takes the form:

$$
d s^{2}=\Delta\left(\frac{2 d x^{0} d x^{1}}{V^{(1)}}+\frac{d x^{2^{2}}}{V^{(2)}}+\frac{d x^{3^{2}}}{V^{(3)}}\right) .
$$

The determinant of the metric is

$$
\operatorname{det} g_{i j}=-\frac{\Delta^{4}}{V^{(1)^{2}} V^{(2)} V^{(3)}} .
$$

The solution for the metric Equation (16) of the Einstein equations in vacuum Equation (14) leads to a degeneration of the type of space: additional commuting Killing vector fields appear, the number of nonignored variables in the metric decreases and, thus, a transition to other types Shapovalov spaces occurs (considered below). Thus, there are no solutions to the Einstein vacuum Equation (14) with a cosmological constant for Shapovalov spaces of type I.

Note that, in modified theories of gravity, it is possible for Shapovalov spaces of type I to have an exact "wave" solution of field equations in vacuum, and this problem requires additional study.

\subsection{Integration of the Eikonal Equation for the Shapovalov Space of Type I}

Separation of variables in the eikonal Equation (3) for the metric Equation (15) gives the eikonal function of the form

$$
\begin{gathered}
\Psi=\lambda_{(0)} x^{0}+\psi_{1}\left(x^{1}\right)+\psi_{2}\left(x^{2}\right)+\psi_{3}\left(x^{3}\right)+F\left(\lambda_{(0)}, \lambda_{(1)}, \lambda_{(2)}\right), \\
\lambda_{(0)}, \lambda_{(1)}, \lambda_{(2)}-\text { const, }
\end{gathered}
$$

where $F\left(\lambda_{(0)}, \lambda_{(1)}, \lambda_{(2)}\right)$ is an arbitrary function of constant separation parameters, and the functions $\psi$ in Equation (18) are defined by the expressions

$$
\begin{gathered}
2 \lambda_{(0)} \psi_{1}=\lambda_{(2)} x^{1}+\lambda_{(1)} \int t_{1}\left(x^{1}\right) d x^{1}, \\
\psi_{2}= \pm \int \sqrt{\lambda_{(1)} t_{2}\left(x^{2}\right)+\lambda_{(2)}} d x^{2}, \quad \psi_{3}= \pm \int \sqrt{\lambda_{(1)} t_{3}\left(x^{3}\right)+\lambda_{(2)}} d x^{3} .
\end{gathered}
$$

\subsection{Integration of the Hamilton-Jacobi Equation of a Test Particle for the Shapovalov Space of Type I}

Separation of variables in the Hamilton-Jacobi equation of a test particle Equation (1) in a privileged coordinate system for the metric Equation (15) gives the complete integral of the action function of a test particle of mass $m$ in the form

$$
\begin{gathered}
S=\lambda_{(0)} x^{0}+\phi_{1}\left(x^{1}\right)+\phi_{2}\left(x^{2}\right)+\phi_{3}\left(x^{3}\right)+F\left(m, \lambda_{(0)}, \lambda_{(1)}, \lambda_{(2)}\right), \\
\lambda_{(0)}, \lambda_{(1)}, \lambda_{(2)}-\text { const }
\end{gathered}
$$

where $F\left(m, \lambda_{(0)}, \lambda_{(1)}, \lambda_{(2)}\right)$ is an arbitrary function of constant parameters, and the functions $\phi$ in Equation (21) are defined by the expressions

$$
2 \lambda_{(0)} \phi_{1}=\lambda_{(2)} x^{1}+\int\left[m^{2} \sigma_{1}\left(x^{1}\right)+\lambda_{(1)} t_{1}\left(x^{1}\right)\right] d x^{1},
$$




$$
\begin{aligned}
& \phi_{2}= \pm \int \sqrt{m^{2} \sigma_{2}\left(x^{2}\right)+\lambda_{(1)} t_{2}\left(x^{2}\right)+\lambda_{(2)}} d x^{2}, \\
& \phi_{3}= \pm \int \sqrt{m^{2} \sigma_{3}\left(x^{3}\right)+\lambda_{(1)} t_{3}\left(x^{3}\right)+\lambda_{(2)}} d x^{3} .
\end{aligned}
$$

\section{Type II Shapovalov Spaces}

Type II Shapovalov spaces admit two Killing vectors and two Killing tensors of the second rank in the complete set and have two subtypes: II.A and II.B. For all types of spaces, below, complete integrals are presented for the eikonal function and for the action function of test particles, and the Einstein equations with the cosmological constant in vacuum are integrated.

\subsection{Type II.A Shapovalov Spaces}

In a privileged coordinate system, the metric can be represented as

$$
g^{i j}=\frac{1}{\Delta}\left(\begin{array}{cccc}
1 & 0 & 0 & 0 \\
0 & 0 & f_{1}\left(x^{1}\right) & 1 \\
0 & f_{1}\left(x^{1}\right) & a_{0}\left(x^{0}\right)+a_{1}\left(x^{1}\right) & 0 \\
0 & 1 & 0 & 0
\end{array}\right)
$$

In the case of conformal Shapovalov spaces, $\Delta$ is an arbitrary function of all variables, and in the case of Shapovalov spaces, the conformal factor is $\Delta=t_{0}\left(x^{0}\right)+t_{1}\left(x^{1}\right)$. Variables $x^{2}$ and $x^{3}$ are ignored, $x^{1}$ is a nonignored isotropic variable.

The Shapovalov spacetime interval of the type II.A can then be written as:

$$
d s^{2}=\Delta\left[d x^{0^{2}}+2 d x^{1} d x^{3}+\frac{1}{a_{0}+a_{1}}\left(d x^{2}-f_{1} d x^{3}\right)^{2}\right] .
$$

The determinant of the metric II.A has the form

$$
g=\operatorname{det} g_{i j}=-\frac{\Delta^{4}}{a_{0}+a_{1}}, \quad a_{0}+a_{1}>0 .
$$

\subsection{Exact Solution of the Einstein Equations for Shapovalov Spaces of Type II.A}

For the metric Equation (25), we obtain the solution of the Einstein vacuum equations with cosmological constant $\Lambda$ (where $x^{1}$ is an isotropic variable):

$$
\begin{gathered}
\Delta=t_{0}\left(x^{0}\right), \quad t_{1}=0, \quad a_{0}=1 / b_{0}\left(x^{0}\right), \quad a_{1}=0, \quad f_{1}=\alpha x^{1}, \quad \alpha-\text { const }, \\
d s^{2}=t_{0}\left[d x^{0^{2}}+2 d x^{1} d x^{3}+b_{0}\left(d x^{2}-\alpha x^{1} d x^{3}\right)^{2}\right], \\
\operatorname{det} g_{i j}=-b_{0} t_{0}{ }^{4}, \quad b_{0}>0 .
\end{gathered}
$$

The functions $b_{0}\left(x^{0}\right)$ and $t_{0}\left(x^{0}\right)$, included in the metric, are determined through the auxiliary function $Y\left(t_{0}\right)$, which is a solution to an ordinary differential equation of the second order:

$$
3 Y \frac{d^{2} Y}{d t_{0}^{2}}-\left(\frac{d Y}{d t_{0}}\right)^{2}-2 \Lambda t_{0}^{2} \frac{d Y}{d t_{0}}-12 \Lambda t_{0} Y+8 \Lambda^{2} t_{0}^{4}=0
$$

where $\Lambda$ is the cosmological constant. 
Then the function $t_{0}\left(x^{0}\right)$ is determined by integrating the relation:

$$
\frac{d t_{0}\left(x^{0}\right)}{d x^{0}}= \pm \sqrt{Y\left(t_{0}\right)}
$$

The function $b_{0}$ is defined through the function $t_{0}$ by the relation:

$$
b_{0}\left(t_{0}\right)=\frac{2 t_{0} Y^{\prime}\left(t_{0}\right)-3 Y\left(t_{0}\right)^{2}-4 \Lambda t_{0}^{3}}{3 \alpha^{2} t_{0}^{2}} .
$$

Note that the order of the differential Equation (31) can be lowered to the first order.

When changing variables of the form

$$
t_{0}=\exp t, \quad Y=X(t) \exp 3 t
$$

the newly obtained differential Equation for $X(t)$ ceases to include the independent variable $t$ and its order can be reduced by introducing a new function $Z(X)=d X(t) / d t$.

The function $Z(X)$, in turn, is a solution to the ordinary differential equation of the first order

$$
3 X Z(X) \frac{d Z(X)}{d X}+Z(X)(-Z(X)+9 X-2 \Lambda)+(3 X-2 \Lambda)(3 X-4 \Lambda)=0 .
$$

For the equation (35), there are particular solutions of the form $Z(X)=\beta X+2 \Lambda$, where $\beta=-3$ or $\beta=-3 / 2$. For these particular solutions, the function $Y\left(t_{0}\right)$ takes the form

$$
Y\left(t_{0}\right)=t_{0}{ }^{3}\left(-2 \Lambda / \beta+\gamma t_{0}{ }^{\beta}\right), \quad \gamma \text { - const. }
$$

For a particular solution (36) at $\beta=-3$, the function $b_{0}\left(x^{0}\right)$ becomes negative, which violates the requirement for the sign of the determinant of the metric. For a particular solution for $\beta=-3 / 2$ the function $b_{0}$ vanishes, which leads to the degeneration of the metric. However, in general, Equations (31) and (35) may have viable solutions.

Ricci tensor and scalar curvature are nonzero and proportional to the cosmological constant $\Lambda$. Weyl tensor and Riemann curvature tensor do not vanish.

\subsection{Integration of the Eikonal Equation for a Shapovalov Space of Type II.A}

Separation of variables in the eikonal equation for the metric of the Shapovalov space of type II.A gives

$$
\begin{gathered}
\Psi=\psi_{0}\left(x^{0}\right)+\psi_{1}\left(x^{1}\right)+\lambda_{(2)} x^{2}+\lambda_{(3)} x^{3}+F\left(\lambda_{(1)}, \lambda_{(2)}, \lambda_{(3)}\right), \\
\lambda_{(1)}, \lambda_{(2)}, \lambda_{(3)}-\mathrm{const}
\end{gathered}
$$

where $F\left(\lambda_{(1)}, \lambda_{(2)}, \lambda_{(3)}\right)$ is an arbitrary function of the separation parameters,

$$
\psi_{0}= \pm \int \sqrt{\lambda_{(1)}-\lambda_{(2)}^{2} a_{0}\left(x^{0}\right)} d x^{0}
$$

and the function $\psi_{1}\left(x^{1}\right)$ is defined by the ordinary differential equation

$$
2\left[\lambda_{(2)} f_{1}\left(x^{1}\right)+\lambda_{(3)}\right] \psi_{1}^{\prime}\left(x^{1}\right)=-\lambda_{(1)}-\lambda_{(2)}^{2} a_{1}\left(x^{1}\right),
$$


where the prime means the ordinary derivative.

4.4. Integration of the Hamilton-Jacobi Equation of a Test Particle for a Shapovalov Space of Type II.A

The complete integral for the action function $S$ of a test particle of mass $m$ takes the form

$$
\begin{gathered}
S=\phi_{0}\left(x^{0}\right)+\phi_{1}\left(x^{1}\right)+\lambda_{(2)} x^{2}+\lambda_{(3)} x^{3}+F\left(m, \lambda_{(1)}, \lambda_{(2)}, \lambda_{(3)}\right) \\
\lambda_{(1)}, \lambda_{(2)}, \lambda_{(3)}-\mathrm{const}
\end{gathered}
$$

where $F\left(m, \lambda_{(1)}, \lambda_{(2)}, \lambda_{(3)}\right)$ is an arbitrary function of the separation parameters,

$$
\phi_{0}= \pm \int \sqrt{m^{2} t_{0}\left(x^{0}\right)-\lambda_{(2)}^{2} a_{0}\left(x^{0}\right)+\lambda_{(1)}} d x^{0},
$$

and the function $\phi_{1}\left(x^{1}\right)$ is determined by the ordinary differential equation

$$
2\left[\lambda_{(2)} f_{1}\left(x^{1}\right)+\lambda_{(3)}\right] \phi_{1}^{\prime}\left(x^{1}\right)=m^{2} t_{1}\left(x^{1}\right)-\lambda_{(2)}^{2} a_{1}\left(x^{1}\right)-\lambda_{(1)} .
$$

\subsection{Type II.B Shapovalov Spaces}

The metric of the Shapovalov II.B space in the privileged coordinate system can be represented as follows:

$$
g^{i j}=\frac{1}{\Delta}\left(\begin{array}{cccc}
1 & 0 & 0 & 0 \\
0 & 0 & f_{1}\left(x^{1}\right) & 1 \\
0 & f_{1}\left(x^{1}\right) & a_{0}\left(x^{0}\right) b_{0}\left(x^{0}\right) & b_{0}\left(x^{0}\right) \\
0 & 1 & b_{0}\left(x^{0}\right) & b_{0}\left(x^{0}\right) / a_{0}\left(x^{0}\right)
\end{array}\right),
$$

moreover, $a_{0} b_{0} \neq 0$. The variable $x^{1}$ is a nonignored isotropic (wave) variable.

The interval II.B is:

$$
d s^{2}=\Delta\left[d x^{0^{2}}+\frac{2 d x^{1}\left(-d x^{2}+a_{0} d x^{3}\right)}{a_{0}-f_{1}}+\frac{a_{0}\left(d x^{2}-f_{1} d x^{3}\right)^{2}}{b_{0}\left(a_{0}-f_{1}\right)^{2}}\right] .
$$

The determinant of the metric II.B has the form

$$
g=\operatorname{det} g_{i j}=-\frac{a_{0} \Delta^{4}}{b_{0}\left(a_{0}-f_{1}\right)^{2}}, \quad a_{0} b_{0}>0 .
$$

When integrating field equations for Shapovalov spaces, functional equations often arise that connect the functions of the metric in different variables.

The Einstein Equation (14) with indices of nonignored variables $\{0,1\}$ can be written for the metric II.B in the form

$$
\partial_{0} \partial_{1}\left(\ln \left[\frac{\Delta}{a_{0}\left(x^{0}\right)-f_{1}\left(x^{1}\right)}\right]\right)=0 .
$$

For the case of Stäckel spaces, when $\Delta=t_{0}\left(x^{0}\right)+t_{1}\left(x^{1}\right)$, we obtain the functional equation:

$$
\partial_{0} \partial_{1}\left(\ln \left[\frac{t_{0}\left(x^{0}\right)+t_{1}\left(x^{1}\right)}{a_{0}\left(x^{0}\right)-f_{1}\left(x^{1}\right)}\right]\right)=0 .
$$


The Equation (49), considered as a functional equation, taking into account the condition $f_{1}{ }^{\prime} \neq 0$ (otherwise the degeneration of the separation type occurs), has the following set of solutions:

$$
\begin{aligned}
& \text { 1. } \Delta=\frac{1}{f_{1}\left(x^{1}\right)+q}-\frac{1}{a_{0}\left(x^{0}\right)+q}, \quad q=\text { const; } \\
& \text { 2. } \Delta=a_{0}\left(x^{0}\right)-f_{1}\left(x^{1}\right) ; \\
& \text { 3. } \Delta=t_{0}\left(x^{0}\right), \quad t_{1}=0, \quad a_{0}=\text { const; } \\
& \text { 4. } \quad \Delta=t_{1}\left(x^{1}\right), \quad t_{0}=0, \quad a_{0}=\text { const. }
\end{aligned}
$$

Note that cases 2, 3 and 4 when solving the Einstein vacuum equations with a cosmological constant Equation (14) lead to contradictions, and only in case 1 we obtain two exact solutions of the field equations, which are listed below.

\subsection{Exact Solution \#1 of Einstein's Equations for II.B Type Shapovalov Space}

The first exact solution of the Einstein vacuum equations for a metric Equation (45) of type II.B in case Equation (50) has the form (where $x^{1}$ is an isotropic wave variable):

$$
\begin{gathered}
a_{0}=a_{0}\left(x^{0}\right), \quad b_{0}=b_{0}\left(x^{0}\right), \quad f_{1}=f_{1}\left(x^{1}\right), \\
d s^{2}=\frac{1}{\left(a_{0}+q\right)\left(f_{1}+q\right)}\left[\left(f_{1}-a_{0}\right) d x^{0^{2}}+2 d x^{1}\left(d x^{2}-a_{0} d x^{3}\right)\right. \\
\left.\quad+\frac{a_{0}}{b_{0}\left(f_{1}-a_{0}\right)}\left(d x^{2}-f_{1} d x^{3}\right)^{2}\right] .
\end{gathered}
$$

The cosmological constant vanishes, the solution has six independent constants:

$$
\Lambda=0, \quad p, q, r, k, \alpha, \beta, \gamma-\mathrm{const}, \quad \gamma=-q \pm \sqrt{\alpha^{2}+\beta^{2}},
$$

Functions included in the conformal factor of the metric, take the form:

$$
\begin{aligned}
t_{0} & =\frac{1-p x^{0^{4}}}{2(q+\gamma)}+r, \quad t_{1}=-\frac{1}{f_{1}\left(x^{1}\right)+q}-r, \\
\Delta & =-\frac{\left(p x^{0^{4}}-1\right) f_{1}\left(x^{1}\right)+p q x^{0^{4}}+q+2 \gamma}{2(q+\gamma)\left(f_{1}\left(x^{1}\right)+q\right)} .
\end{aligned}
$$

The functions $a_{0}\left(x^{0}\right), b_{0}\left(x^{0}\right)$ and $f_{1}\left(x^{1}\right)$, included in the metric, are defined by the relations:

$$
\begin{gathered}
f_{1}\left(x^{1}\right)=\alpha \cos \left(k x^{1}\right)+\beta \sin \left(k x^{1}\right)+\gamma, \\
a_{0}\left(x^{0}\right)=\frac{q\left(p x^{0^{4}}+1\right)+2 \gamma}{1-p x^{0^{4}}}, \\
\Delta g^{23}=b_{0}\left(x^{0}\right)=-\frac{k^{2}\left(p x^{0^{4}}-1\right)\left(q\left(p x^{0^{4}}+1\right)+2 \gamma\right)}{16 p x^{0^{2}}},
\end{gathered}
$$




$$
\begin{gathered}
\Delta g^{22}=a_{0} b_{0}=\frac{k^{2}\left(q\left(p x^{0^{4}}+1\right)+2 \gamma\right)^{2}}{16 p x^{0^{2}}}, \\
\Delta g^{33}=b_{0} / a_{0}=\frac{k^{2}\left(p x^{0^{4}}-1\right)^{2}}{16 p x^{0^{2}}},
\end{gathered}
$$

The obtained metric depends on the wave variable $x^{1}$ through harmonic functions.

The determinant of the metric is:

$$
\operatorname{det} g_{i j}=-\frac{p x^{0^{2}}\left(\left(p x^{0^{4}}-1\right) f_{1}\left(x^{1}\right)+q\left(p x^{0^{4}}+1\right)+2 \gamma\right)^{2}}{k^{2}(q+\gamma)^{4}\left(f_{1}\left(x^{1}\right)+q\right)^{4}}, \quad p>0,
$$

For this solution, the Ricci tensor $R_{i j}$, the scalar curvature $R$ and the cosmological constant $\Lambda$ vanish. The Riemann curvature tensor and the Weyl tensor do not vanish.

\subsection{Exact Solution \#2 of Einstein's Equations for Shapovalov Space of Type II.B}

The second exact solution of Einstein's vacuum equations for metric Equation (45) in case Equation (50) has four independent constant parameters, the cosmological constant vanishes:

$$
\Lambda=0, \quad p, q, r, k-\text { const }, \quad p q<0,
$$

Apart from the conformal factor $\Delta\left(x^{0}, x^{1}\right)$, the metric is determined by three functions (here the variable $x^{1}$ is an ignored isotropic variable):

$$
a_{0}=a_{0}\left(x^{0}\right), \quad b_{0}=b_{0}\left(x^{0}\right), \quad f_{1}=f_{1}\left(x^{1}\right) .
$$

The spacetime interval takes the form:

$$
\begin{gathered}
d s^{2}=\frac{1}{\left(a_{0}+q\right)\left(f_{1}+q\right)}\left[\left(f_{1}-a_{0}\right) d x^{0^{2}}+2 d x^{1}\left(d x^{2}-a_{0} d x^{3}\right)\right. \\
\left.+\frac{a_{0}}{b_{0}\left(f_{1}-a_{0}\right)}\left(d x^{2}-f_{1} d x^{3}\right)^{2}\right] .
\end{gathered}
$$

The functions included in the conformal factor of the metric $\Delta$ are determined by the expressions:

$$
t_{0}\left(x^{0}\right)=\frac{x^{0^{4}}}{p}-k, \quad t_{1}\left(x^{1}\right)=k-\frac{1}{q x^{1^{2}}}, \quad \Delta\left(x^{0}, x^{1}\right)=\frac{x^{0^{4}}}{p}-\frac{1}{q x^{1^{2}}},
$$

The functions $a_{0}\left(x^{0}\right), b_{0}\left(x^{0}\right)$ and $f_{1}\left(x^{1}\right)$ and the components of the metric are determined by the relations:

$$
\begin{gathered}
f_{1}\left(x^{1}\right)=q x^{1^{2}}-r, \\
a_{0}\left(x^{0}\right)=\frac{p}{x^{0^{4}}}-r, \\
\Delta g^{23}=b_{0}\left(x^{0}\right)=\frac{q}{4 p} x^{0^{2}}\left(r x^{0^{4}}-p\right),
\end{gathered}
$$




$$
\begin{gathered}
\Delta g^{22}=a_{0}\left(x^{0}\right) b_{0}\left(x^{0}\right)=-\frac{q\left(p-r x^{0^{4}}\right)^{2}}{4 p x^{0^{2}}}, \\
\Delta g^{33}=a_{0}\left(x^{0}\right) / b_{0}\left(x^{0}\right)=-\frac{4 p}{q x^{0^{6}}} \\
f_{1}\left(x^{1}\right)-a_{0}\left(x^{0}\right)=q x^{1^{2}}-\frac{p}{x^{0^{4}}} .
\end{gathered}
$$

The determinant of the metric takes the form:

$$
g=\operatorname{det} g_{i j}=\frac{4 x^{0^{2}}\left(p-q x^{0^{4}} x^{1^{2}}\right)^{2}}{p^{3} q^{5} x^{1^{8}}}, \quad p q<0,
$$

For a given exact solution, the Ricci tensor, scalar curvature and cosmological constant vanish. The Riemann curvature tensor and the Weyl tensor do not vanish.

\subsection{Integration of the Eikonal Equation for the Shapovalov Space of Type II.B}

Separation of variables in the eikonal Equation (3) for a Shapovalov space of type II.B in a privileged coordinate system with metric Equation (45) gives the following form of the eikonal function

$$
\Psi=\psi_{0}\left(x^{0}\right)+\psi_{1}\left(x^{1}\right)+\lambda_{(2)} x^{2}+\lambda_{(3)} x^{3}, \quad \lambda_{(1)}, \lambda_{(2)}, \lambda_{(3)}-\text { const },
$$

where the functions $\psi_{0}$ and $\psi_{0}$ are defined by the relations

$$
\begin{gathered}
\psi_{0}= \pm \int \sqrt{\lambda_{(1)}-\frac{a_{0}\left(x^{0}\right)}{b_{0}\left(x^{0}\right)}\left(\lambda_{(2)} a_{0}\left(x^{0}\right)+\lambda_{(3)}\right)^{2}} d x^{0}, \\
\psi_{1}=-\frac{\lambda_{(1)}}{2} \int \frac{d x^{1}}{\lambda_{(2)} f_{1}\left(x^{1}\right)+\lambda_{(3)}} .
\end{gathered}
$$

4.9. Integration of the Hamilton-Jacobi Equation of Motion of a Test Particle for the Shapovalov Space of Type II.B

Separation of variables in the Hamilton-Jacobi Equation (1) for Shapovalov spaces of type II.B with metric Equation (45) in a privileged coordinate system gives the full integral of the action function of a test particle of mass $m$ of the form:

$$
S=\phi_{0}\left(x^{0}\right)+\phi_{1}\left(x^{1}\right)+\lambda_{(2)} x^{2}+\lambda_{(3)} x^{3}, \quad \lambda_{(1)}, \lambda_{(2)}, \lambda_{(3)}-\text { const },
$$

where the functions $\phi_{0}$ and $\phi_{1}$ are defined by the following relations:

$$
\begin{gathered}
\phi_{0}= \pm \int \sqrt{m^{2} t_{0}\left(x^{0}\right)+\lambda_{(1)}-\frac{a_{0}\left(x^{0}\right)}{b_{0}\left(x^{0}\right)}\left(\lambda_{(2)} a_{0}\left(x^{0}\right)+\lambda_{(3)}\right)^{2}} d x^{0} \\
\phi_{1}=\frac{1}{2} \int \frac{m^{2} t_{1}\left(x^{1}\right)-\lambda_{(1)}}{\lambda_{(2)} f_{1}\left(x^{1}\right)+\lambda_{(3)}} d x^{1} .
\end{gathered}
$$

In case when the function $f_{1}$ is a constant, it can be set equal to zero by transformation of coordinates. 


\section{Type III Shapovalov Spaces}

Type III Shapovalov spaces admit three Killing vectors and one Killing tensor of the second rank in a complete set. Metric of a Shapovalov space of type III in the privileged coordinate system can be written as follows:

$$
g^{i j}=\frac{1}{\Delta}\left(\begin{array}{cccc}
0 & 1 & g^{02}\left(x^{0}\right) & g^{03}\left(x^{0}\right) \\
1 & 0 & 0 & 0 \\
g^{02}\left(x^{0}\right) & 0 & g^{22}\left(x^{0}\right) & g^{23}\left(x^{0}\right) \\
g^{03}\left(x^{0}\right) & 0 & g^{23}\left(x^{0}\right) & g^{33}\left(x^{0}\right)
\end{array}\right)
$$

In the case of Shapovalov spaces, the conformal factor can be set equal to one $(\Delta=1)$, and in the case of conformal Shapovalov spaces, the conformal factor $\Delta$ is an arbitrary function of all four variables.

The metric interval Equation (80) can be written in the following form $(p, q=2,3)$ :

$$
d s^{2}=\Delta\left[2 d x^{0} d x^{1}+g_{p q}\left(x^{0}\right)\left(d x^{p}+g^{(p)}\left(x^{0}\right) d x^{1}\right)\left(d x^{q}+g^{(q)}\left(x^{0}\right) d x^{1}\right)\right] .
$$

The last expression, disregarding the conformal factor $\Delta$, contains five arbitrary functions of one variable $x^{0}$, as in the metric Equation (80). Note that the metric Equation (81) is known in the literature as the metric of a gravitational wave (see [1]).

\subsection{Exact Solution of the Einstein Equations for Shapovalov Spaces Type III}

Integration of Einstein's equations in vacuum for the metric Equation (81) leads to an exact solution (gravitational wave), which can be represented as

$$
d s^{2}=2 d x^{0} d x^{1}-\exp \left(-\gamma_{0}\right)\left(\exp \beta_{0} \cosh \alpha_{0} d x^{2^{2}}+\exp \left(-\beta_{0}\right) \cosh \alpha_{0} d x^{3^{2}}+2 \sinh \alpha_{0} d x^{2} d x^{3}\right)
$$

and only two functions are independent, the function $\beta_{0}$ can be expressed in terms of the rest

$$
\beta_{0}=\int \frac{\sqrt{2 \gamma_{0}^{\prime \prime}-\gamma_{0}^{\prime 2}-\alpha_{0}^{\prime 2}}}{\cosh \alpha_{0}} d x^{0}, \quad \Lambda=0
$$

where $\alpha_{0}$ and $\gamma_{0}$ are arbitrary functions of one variable $x^{0}$ (wave variable), and the prime means ordinary differentiation with respect to $x^{0}$.

\subsection{Integration of the Eikonal Equation for the Shapovalov Space Type III}

Separation of variables in the eikonal Equation (3) for the metric Equation (80) gives the complete integral for the eikonal function of the form

$$
\begin{gathered}
\Psi=\psi_{0}\left(x^{0}\right)+\lambda_{(1)} x^{1}+\lambda_{(2)} x^{2}+\lambda_{(3)} x^{3}+F\left(\lambda_{(1)}, \lambda_{(2)}, \lambda_{(3)}\right), \\
\lambda_{(1)}, \lambda_{(2)}, \lambda_{(3)}-\mathrm{const}
\end{gathered}
$$

where $F\left(\lambda_{(1)}, \lambda_{(2)}, \lambda_{(3)}\right)$ is an arbitrary function of parameters, and the function $\psi_{0}\left(x^{0}\right)$ in Equation (84) is defined by the following expression

$$
\psi_{0}\left(x^{0}\right)=-\frac{1}{2} \int \frac{\lambda_{(p)} \lambda_{(q)} g^{p q}\left(x^{0}\right)}{\lambda_{(1)}+\lambda_{(p)} g^{0 p}\left(x^{0}\right)} d x^{0}, \quad p, q=2,3 .
$$


When the components of the metric $g^{0 p}$ become constants, they can be converted to zero by transforming the coordinates.

\subsection{Integration of the Hamilton-Jacobi Equation of a Test Particle for Shapovalov Space Type III}

Separation of variables in the Hamilton-Jacobi equation of a test particle Equation (1) for the metric Equation (80) gives a complete integral for the action function of a test particle of mass $m$ of the form

$$
\begin{gathered}
S=\phi_{0}\left(x^{0}\right)+\lambda_{(1)} x^{1}+\lambda_{(2)} x^{2}+\lambda_{(3)} x^{3}+F\left(m, \lambda_{(1)}, \lambda_{(2)}, \lambda_{(3)}\right) \\
\lambda_{(1)}, \lambda_{(2)}, \lambda_{(3)}-\mathrm{const}
\end{gathered}
$$

where $F\left(m, \lambda_{(1)}, \lambda_{(2)}, \lambda_{(3)}\right)$ is an arbitrary function of parameters, and the function $\phi_{0}\left(x^{0}\right)$ in Equation (86) is defined by the following expression

$$
\phi_{0}\left(x^{0}\right)=\frac{1}{2} \int \frac{m^{2}-\lambda_{(p)} \lambda_{(q)} g^{p q}\left(x^{0}\right)}{\lambda_{(1)}+\lambda_{(p)} g^{0 p}\left(x^{0}\right)} d x^{0}, \quad p, q=2,3 .
$$

Note that the variables $x^{0}$ and $x^{1}$ of the privileged coordinate system are isotropic variables, and the spacetime metric depends only on one wave variable $x^{0}$ and is interpreted as an exact solution for a gravitational wave.

\section{Conclusions}

The paper presents an effective mathematical tool for obtaining and analyzing wave-like models of spaces in the theory of gravity. Namely, a classification is carried out and all possible types of spacetime models are listed, allowing separation of variables in the eikonal equation and the Hamilton-Jacobi equation of test particles with separation of isotropic (null) wave variables. We call such spaces wave-like Shapovalov spaces. The classification is based on the type of "complete set" of Killing vectors and Killing tensors admitted by the corresponding type of the considered spaces. The existence of such a "complete set" in the considered spacetime models determines the possibility of complete separation of variables.

The types of spaces obtained in this work are "wave-like", i.e., lead to solutions of field equations of the "gravitational wave" type and, thus, are of interest for gravitational-wave astronomy. Since the proposed method is applicable to various modified theories of gravity, it can be used to carry out a comparative analysis of the same type of gravitational-wave solutions in various modified theories of gravity in order to select viable theories (according to observational data of gravitational wave astronomy) that claim to describe the phenomena of "dark energy" and "dark matter".

As an example of the effectiveness of the proposed method, exact wave solutions of the Einstein equations with a cosmological constant in vacuum are obtained. Solutions are written in coordinate systems where variables are separated. Particular cases of the models under consideration in Einstein's theory of gravity are exact solutions of the "gravitational wave" type of Robinson, Bondi, Trautman, Peres.

The models of spaces proposed in the work allow integration in quadratures of the equations of motion of test particles and radiation, which is significant for the theoretical foundations and methods of gravitational-wave astronomy. As an example of the application of the considered approach, for all types of spaces under consideration, the form of a complete integral for the action function of test particles and for the eikonal function that determines the propagation of radiation is obtained. This makes it possible for the considered models to describe in quadratures the motion of test particles. 
Author Contributions: Conceptualization and methodology, K.O.; investigation, K.O. and E.O.; validation, K.O. and E.O.; writing-original draft preparation, K.O. and E.O.; writing-review and editing, K.O.; funding acquisition, K.O. All authors have read and agreed to the published version of the manuscript.

Funding: The reported study was funded by RFBR, project number N 20-01-00389 A.

Conflicts of Interest: The authors declare no conflict of interest.

\section{References}

1. Landau, L.; Lifshitz, E. The Classical Theory of Fields, 4th ed.; Course of Theoretical Physics Series; Butterworth-Heinemann: Oxford, UK, 1975; Volume 2, p. 402.

2. Stäckel, P. Ueber die integration der Hamilton'schen differentialgleichung mittelst separation der variabeln. Math. Ann. 1897, 49, 145-147. [CrossRef]

3. Bagrov, V.; Meshkov, A.; Shapovalov, V.; Shapovalov, A. Separation of variables in the Klein-Gordon equation. I. Sov. Phys. J. 1973, 16, 1533-1538. [CrossRef]

4. Naiminov, A.; Shapovalov, V. Symmetry of the Hamilton-Jacobi and Klein-Gordon equations. Sov. Phys. J. 1974, 17, 571-572. [CrossRef]

5. Shapovalov, V. Symmetry of equations of motion for a free particle in Riemann space. Sov. Phys. J. 1975, 18, 1650-1654. [CrossRef]

6. Shapovalov, V. Symmetry and separation of variables in Hamilton-Jacobi equations. I. Sov. Phys. J. 1978, 21, 1124-1129. [CrossRef]

7. Shapovalov, V. Symmetry and separation of variables in Hamilton-Jacobi equations. II. Sov. Phys. J. 1978, 21, 1130-1132. [CrossRef]

8. Shapovalov, V. Symmetry and integration of the Hamilton-Jacobi equation for a free particle in a Riemannian space. Sov. Phys. J. 1978, 21, 718-721. [CrossRef]

9. Shapovalov, V. Stäckel spaces. Sib. Math. J. 1979, 20, 790-800. [CrossRef]

10. Obukhov, V.; Osetrin, K. Variables separation in gravity. In Proceedings of the Fourth International Winter Conference on Mathematical Methods in Physics (WC2004), Rio de Janeiro, Brazil, 9-13 August 2004; Volume 13; pp. 127-133. [CrossRef]

11. Benenti, S. Separability in Riemannian manifolds. Methods Appl. 2016, 12. [CrossRef]

12. Bagrov, V.; Obukhov, V.; Osetrin, K. Classification of null-Stäckel electrovac metrics with cosmological constant. Gen. Relativ. Gravit. 1988, 20, 1141-1154. [CrossRef]

13. Osetrin, K.; Obukhov, V.; Filippov, A. Homogeneous spacetimes and separation of variables in the Hamilton-Jacobi equation. J. Phys. A Math. Gen. 2006, 39, 6641-6647. [CrossRef]

14. Obukhov, V.; Osetrin, K.; Filippov, A. Metrics of homogeneous spaces admitting (3.1)-type complete sets. Russ. Phys. J. 2002, 45, 42-48. [CrossRef]

15. Makarenko, A.; Osetrin, K. Conformally Steckel metrics in Einstein spaces. Russ. Phys. J. 1999, 42, 889-896. [CrossRef]

16. Bagrov, V.; Obukhov, V.; Osetrin, K. Nontrivial conformally Stäckel metrics in Einstein spaces. Russ. Phys. J. 1997, 40, 995-999. [CrossRef]

17. Bagrov, V.; Istomin, A.; Obukhov, V.; Osetrin, K. Classification of conformal Stäckel spaces in the Vaidia problem. Russ. Phys. J. 1996, 39, 744-749. [CrossRef]

18. Bagrov, V.; Obukhov, V.; Osetrin, K. Isotropic conformal Stäckel metrics of conformally flat spaces. Russ. Phys. J. 1998, 41, 1149-1154. [CrossRef]

19. Osetrin, K.; Filippov, A.; Osetrin, E. Models of generalized scalar-tensor gravitation theories with radiation allowing the separation of variables in the eikonal equation. Russ. Phys. J. 2018, 61, 1383-1391. [CrossRef]

20. Osetrin, E.; Osetrin, K.; Filippov, A. Plane gravitational waves in spatially-homogeneous models of type-(3.1) Stäckel spaces. Russ. Phys. J. 2019, 62, 292-301. [CrossRef]

21. Osetrin, K.; Filippov, A.; Osetrin, E. The spacetime models with dust matter that admit separation of variables in Hamilton-Jacobi equations of a test particle. Modern Phys. Lett. A 2016, 31. [CrossRef] 
22. Osetrin, E.; Osetrin, K. Pure radiation in space-time models that admit integration of the eikonal equation by the separation of variables method. J. Math. Phys. 2017, 58. [CrossRef]

23. Osetrin, E.; Osetrin, K.; Filippov, A. Spatially homogeneous models Stäckel spaces of type (2.1). Russ. Phys. J. 2020, 63, 410-419. [CrossRef]

24. Osetrin, E.; Osetrin, K.; Filippov, A. Spatially homogeneous conformally Stäckel spaces of type (3.1). Russ. Phys. J. 2020, 63, 403-409. [CrossRef]

25. Obukhov, V. Hamilton-Jacobi equation for a charged test particle in the Stäckel space of type (2.0). Symmetry 2020, 12, 1289. [CrossRef]

26. Nojiri, S.; Odintsov, S.; Oikonomou, V. Modified gravity theories on a nutshell: Inflation, bounce and late-time evolution. Phys. Rep. 2017, 692,1-104. [CrossRef]

27. Capozziello, S.; de Laurentis, M. Extended theories of gravity. Phys. Rep. 2011, 509, 167-321. [CrossRef]

28. Capozziello, S.; Francaviglia, M. Extended theories of gravity and their cosmological and astrophysical applications. Gen. Relativ. Gravit. 2008, 40, 357-420. [CrossRef]

29. Nojiri, S.; Odintsov, S. Unified cosmic history in modified gravity: From $F(R)$ theory to Lorentz non-invariant models. Phys. Rep. 2011, 505, 59-144. [CrossRef]

30. Nojiri, S.; Odintsov, S. Introduction to modified gravity and gravitational alternative for dark energy. Int. J. Geom. Methods Mod. Phys. 2007, 4, 115-145. [CrossRef]

31. Bamba, K.; Capozziello, S.; Nojiri, S.; Odintsov, S. Dark energy cosmology: The equivalent description via different theoretical models and cosmography tests. Astrophys. Space Sci. 2012, 342, 155-228. [CrossRef]

(C) 2020 by the authors. Licensee MDPI, Basel, Switzerland. This article is an open access article distributed under the terms and conditions of the Creative Commons Attribution (CC BY) license (http:/ / creativecommons.org/licenses/by/4.0/). 